\title{
Regularized overlap and the chiral determinant
}

\author{
C.D. Fosco ${ }^{*}$ and R.C. Trinchero ${ }^{\dagger}$ \\ Centro Atómico Bariloche 8400 S.C. de Bariloche, Argentina
}

December 27, 2018

\begin{abstract}
We study the relationship between the continuum overlap and its corresponding chiral determinant, showing that the former amounts to an unregularised version of the latter. We then construct a regularised continuum overlap, and consider the chiral anomalies that follow therefrom. The relation between these anomalies and the ones derived from the formal (i.e., unregularised) overlap is elucidated.
\end{abstract}

\section{Introduction.}

Chiral Gauge Theories [1], namely, the ones where the left and right components of a fermionic field couple asymmetrically to a gauge field are of fundamental importance in High Energy Physics.

Their study is of relevance, for example, to understand the dynamics of the Standard Model, where their characteristic properties have important consequences, such as the anomaly cancelation conditions.

To find a proper definition of the effective action for a fermionic field in the presence of an external gauge field is usually the first step towards a full quantization of the theory. This step is equivalent to the definition (if possible beyond the perturbative level) of a chiral determinant. Many attempts

\footnotetext{
* Member of CONICET, Argentina.

${ }^{\dagger}$ Member of Instituto Balseiro and CONICET, Argentina.
} 
have been done to achieve this goal, and in recent years some new proposals emerged, which, in spite of being at first sight different, are related. They share the property of introducing an infinite number of fermionic fields for each point in spacetime. This appears as an extra dimension in Kaplan's formulation [2], as an infinite number of Pauli-Villars fields in Slavnov's proposal [3, 4], and as a continuum of fermionic degrees of freedom living inside each lattice cell in t'Hooft approach [5].

The overlap formulation [6] (inspired in an earlier idea of Kaplan [2]) seems to correctly define a fermionic chiral determinant when constructed on a lattice. In this letter we study the overlap in the continuum, showing explicitly that it is equivalent to a non-regularised version of its corresponding chiral determinant. For the particular case of the modulus of the chiral determinant in two spacetime dimensions it yields a finite result.

It has been shown $(\llbracket 7,8])$ that the formal (i.e., unregularized) overlap in the continuum yields anomalies when its gauge variation is calculated in a perturbative approach. A similar calculation has been done on the lattice ( cutoff tends to zero.

We show that the anomalies derived from the unregularised overlap may lead to ambiguous results when calculating the covariant divergence of the chiral current in terms of the full fermion propagator in the presence of an external gauge field, and then define a properly regularised continuum overlap, which leads to consistent anomalies.

\section{Definitions and conventions.}

We shall consider the overlap associated to a chiral Dirac operator $\not D_{L}=\not D P_{L}$, where $\not D=\gamma_{\mu}\left(\partial_{\mu}+A_{\mu}\right), P_{L}=\frac{1+\gamma_{5}}{2}$ is the left chirality projector, and Dirac's matrices are chosen according to the representation:

$$
\gamma_{\mu}=\left(\begin{array}{cc}
0 & \sigma_{\mu}^{\dagger} \\
\sigma_{\mu} & 0
\end{array}\right), \gamma_{5}=\left(\begin{array}{cc}
1 & 0 \\
0 & -1
\end{array}\right), \sigma_{\mu} \stackrel{d=4}{=}(\vec{\sigma}, 1), \sigma_{\mu} \stackrel{d=2}{=}(1, i)
$$

where $\vec{\sigma}$ denote the three familiar Pauli's matrices. $A_{\mu}(x)$ is a (anti-hermitian) gauge connection for the gauge group $\mathcal{G}$. It may be written as $A_{\mu}(x)=$ $A_{\mu}^{a}(x) \tau_{a}$, with real components $A_{\mu}^{a}(x), \tau_{a}^{\dagger}=-\tau_{a}$, and $\left[\tau_{a}, \tau_{b}\right]=f_{a b c} \tau_{c}$. 
The overlap $\mathcal{O}$ corresponding to $\not_{L}$ is defined, following Narayanan and Neuberger, by

$$
\mathcal{O}=\frac{\langle+\mid A+\rangle}{|\langle+\mid A+\rangle|} \frac{\langle A+\mid A-\rangle}{\langle+\mid-\rangle} \frac{\langle A-\mid-\rangle}{|\langle A-\mid-\rangle|},
$$

where $|A \pm\rangle$ are the Dirac vacua corresponding to the Hamiltonians

$$
\mathcal{H}_{ \pm}=\gamma_{5}(\not D \pm M)=\left(\begin{array}{cc} 
\pm M & -C^{\dagger} \\
-C & \mp M
\end{array}\right),
$$

respectively, and $| \pm\rangle=\left.|A \pm\rangle\right|_{A=0}$. The operator $C$ is defined by $C=\sigma_{\mu}\left(\partial_{\mu}+\right.$ $A_{\mu}$ ). Scalar products between the different Dirac vacua appearing in (2) may be put in terms of Slater determinants built from negative energy eigenspinors of the corresponding first-quantized Hamiltonians [6]. These negative energy eigenspinors satisfy

$$
\mathcal{H}_{ \pm}\left|v_{ \pm}(\lambda)\right\rangle=-\omega(\lambda)\left|v_{ \pm}(\lambda)\right\rangle \quad \omega(\lambda)>0,
$$

with the index $\lambda$ labeling the eigenstates t.

The energy $\omega(\lambda)$ is independent of the sign of $M$, and normalized negative energy eigenstates can be constructed as follows 2

$$
\left|v_{ \pm}(\lambda)\right\rangle=\sqrt{\frac{\omega(\lambda) \mp M}{2 \omega(\lambda)}}\left(\begin{array}{c}
\left|v_{1}(\lambda)\right\rangle \\
\frac{C}{\omega(\lambda) \mp M}\left|v_{1}(\lambda)\right\rangle
\end{array}\right),
$$

where $\left|v_{1}(\lambda)\right\rangle$ are orthonormal eigenfunctions of the positive hermitian operator $C^{\dagger} C$, satisfying

$$
C^{\dagger} C\left|v_{1}(\lambda)\right\rangle=\left(\omega^{2}(\lambda)-M^{2}\right)\left|v_{1}(\lambda)\right\rangle .
$$

The number of components of $\left|v_{1}(\lambda)\right\rangle$ is half the one of $\left|v_{ \pm}(\lambda)\right\rangle$. Of course, the eigenfunctions $\left|v_{ \pm}(\lambda)\right\rangle$ are not uniquely determined, since their phases

\footnotetext{
${ }^{1}$ We use the same ket symbol $\mid>$ to denote second-quantization vacua and firstquantization eigenspinors, since no confussion should arise.

${ }^{2}$ We shall assume at this stage that there are no zero modes either for $C$ or $C^{\dagger}$. Had zero modes been present, the chiral determinant would have been equal to zero.
} 
may be changed without affecting the normalisation. It will turn out, however, that (5) is a convenient choice since it makes the factor $\frac{\langle A+\mid A-\rangle}{\langle+\mid-\rangle}$ in (2) real, while the remaining two factors are pure phases. This makes the identification of the modulus and phase of $\mathcal{O}$ easier.

As a straightforward calculation shows, the relevant scalar products in (2) may be put in terms of the above-defined eigenspinors as

$$
\langle A+\mid A-\rangle=\operatorname{det}_{\lambda, \lambda^{\prime}}\left\langle v_{+}(\lambda) \mid v_{-}\left(\lambda^{\prime}\right)\right\rangle,\langle \pm \mid A \pm\rangle=\operatorname{det}_{\lambda, \lambda^{\prime}}\left\langle v_{ \pm}^{0}(\lambda) \mid v_{ \pm}\left(\lambda^{\prime}\right)\right\rangle,
$$

where $\left|v_{ \pm}^{0}(\lambda)\right\rangle$ denotes the negative-energy eigenfunction $\left|v_{ \pm}(\lambda)\right\rangle$ for $A_{\mu}=0$.

According to the overlap formalism, the effective action following from $\mathcal{O}$ should coincide, when $M \rightarrow \infty$, with the normalized chiral Dirac operator effective action, i.e.,

$$
\lim _{M \rightarrow \infty} \Gamma_{\mathcal{O}}(A, M) \equiv-\lim _{M \rightarrow \infty} \log \mathcal{O}(A, M)=-\log \operatorname{det}\left(\not \partial_{L}^{-1} \not D_{L}\right) \equiv \Gamma_{L}(A)
$$

It is convenient to split $\Gamma_{\mathcal{O}}$ into its real and imaginary parts, since they enjoy very different properties (akin to the ones of the real and imaginary parts of $\left.\Gamma_{L}\right)$. The real part of $\Gamma_{\mathcal{O}}$ comes from the factor $\frac{\langle A+\mid A-\rangle}{\langle+\mid-\rangle}$. By using (7), (6) and (5) it is possible to write $\langle A+\mid A-\rangle$ as the determinant of an operator acting on the Hilbert space generated by the set $\left\{\left|v_{1}(\lambda)\right\rangle\right\}$ of eigenfunctions of $C^{\dagger} C$

$$
\langle A+\mid A-\rangle=\operatorname{det}\left(\frac{C^{\dagger} C}{C^{\dagger} C+M^{2}}\right)^{1 / 2} \text {. }
$$

Then

$$
\begin{gathered}
\operatorname{Re} \Gamma_{\mathcal{O}}=-\operatorname{Re} \log \left[\frac{\langle A+\mid A-\rangle}{\langle+\mid-\rangle}\right] \\
=-\frac{1}{2} \operatorname{Tr} \log \left[\frac{C^{\dagger} C}{C^{\dagger} C+M^{2}}\right]+\frac{1}{2} \operatorname{Tr} \log \left[\frac{C_{0}^{\dagger} C_{0}}{C_{0}^{\dagger} C_{0}+M^{2}}\right],
\end{gathered}
$$

where, of course, the functional trace is also over the Hilbert space generated by $\left\{\left|v_{1}(\lambda)\right\rangle\right\}$. The formal $M \rightarrow \infty$ limit of (10) is

$$
\operatorname{Re} \Gamma_{\mathcal{O}} \rightarrow-\frac{1}{2} \operatorname{Tr} \log \left(\frac{C^{\dagger} C}{M^{2}}\right)+\frac{1}{2} \operatorname{Tr} \log \left(\frac{C_{0}^{\dagger} C_{0}}{M^{2}}\right)
$$


which may also be put in terms of Dirac operators as

$$
\operatorname{Re} \Gamma_{\mathcal{O}} \rightarrow-\frac{1}{2} \operatorname{Tr} \log \left(\not D_{L}^{\dagger} \not D_{L}\right)-\frac{1}{2} \operatorname{Tr} \log \left(\partial_{L}^{\dagger} \partial_{L}\right) .
$$

which is, of course, unregularized. To see the role played by $M$, we note that, keeping it finite, $\operatorname{Re} \Gamma_{\mathcal{O}}$ may be written as

$$
\begin{gathered}
\operatorname{Re} \Gamma_{\mathcal{O}}(A, M)=-\frac{1}{2} \sum_{s=0}^{1} C_{s} \operatorname{Tr} \log \left(C^{\dagger} C+M_{s}^{2}\right) \\
+\frac{1}{2} \sum_{s=0}^{1} C_{s} \operatorname{Tr} \log \left(C_{0}^{\dagger} C_{0}+M_{s}^{2}\right),
\end{gathered}
$$

where $C_{0}=1, C_{1}=-1, M_{0}=0$ and $M_{1}=M$. This form makes it explicit the fact that the finite- $M$ overlap in two spacetime dimensions yields a regularized real part of the effective action, since it corresponds to a PauliVillars like regularization, with $M$ playing the role of the regulator mass. Moreover, this regularization is gauge invariant, since it is provided by an operator depending on $C^{\dagger} C$, which transforms in a gauge covariant way. It also follows from (13) that the real part of the overlap effective action is not regularized in more than two dimensions, since more Pauli-Villars regulators would be required.

Proceeding analogously to the case of the real part, it is possible to write the imaginary part of the overlap effective action as the functional trace (over the same functional space) of an operator:

$$
\operatorname{Im} \Gamma_{\mathcal{O}}=\frac{1}{2 i} \operatorname{Tr}\left[\log \left(\frac{\mathcal{C}^{\dagger} \mathcal{C}_{0}}{\left(1+\mathcal{C}^{\dagger} \mathcal{C}_{0}\right)^{2}}\right)-\log \left(\frac{\mathcal{C}_{0}^{\dagger} \mathcal{C}}{\left(1+\mathcal{C}_{0}^{\dagger} \mathcal{C}\right)^{2}}\right)\right]=\frac{1}{2 i}\left(A-A^{*}\right)
$$

where

$$
\begin{aligned}
A & =\operatorname{Tr}\left[\log \left(\mathcal{C}^{\dagger} \mathcal{C}_{0}\left(1+\mathcal{C}^{\dagger} \mathcal{C}_{0}\right)^{-2}\right]\right. \\
\mathcal{C} & =C(\hat{\omega}+M)^{-1} \quad \hat{\omega}=\left(C^{\dagger} C+M^{2}\right)^{\frac{1}{2}} \\
\mathcal{C}_{0} & =C_{0}\left(\hat{\omega}_{0}+M\right)^{-1} \quad \hat{\omega}_{0}=\left(C_{0}^{\dagger} C_{0}+M^{2}\right)^{\frac{1}{2}} .
\end{aligned}
$$

As a check, one can consider the formal limit $M \rightarrow \infty$

$$
\operatorname{Im} \Gamma_{\mathcal{O}} \stackrel{M \rightarrow \infty}{=} \frac{1}{2 i} \operatorname{Tr}\left[\log \left(\frac{C^{\dagger} C_{0}}{4 M^{2}}\right)-\log \left(\frac{C_{0}^{\dagger} C}{4 M^{2}}\right)\right]
$$


which, at the unregularized level, is a sensible definition of the imaginary part of the effective action for the corresponding chiral determinant. It is evident from (14) that $\operatorname{Im} \Gamma_{\mathcal{O}}$ has the following structure:

$$
\operatorname{Im} \Gamma_{\mathcal{O}}=\operatorname{Im} \operatorname{Tr} F\left(\mathcal{C}^{\dagger} \mathcal{C}_{0}\right)
$$

with $F(x)=\frac{x}{(1+x)^{2}}$. This functional form of $F$ is not accidental, as the following argument shows: From the definition (2) of the overlap it follows that $\operatorname{Im} \Gamma$ is odd in $M$,

$$
\operatorname{Im} \Gamma_{\mathcal{O}}(A,-M)=-\operatorname{Im} \Gamma_{\mathcal{O}}(A, M) .
$$

When this condition is impossed on (17) (assuming $F$ real), it requires for $F$ to satisfy the functional equation

$$
F\left(\frac{1}{x}\right)=F(x)
$$

condition which is satisfied by $F(x)=\frac{x}{(1+x)^{2}}$, but not by an arbitrary function. This very same property is indeed imcompatible with a PauliVillars like regularization of the imaginary part, since, as it will be shown in the next section, that regularization would require, for example, in two dimensions, a functional form

$$
F(x)=\frac{x}{1+x},
$$

which fails to satisfy (19). We have concluded that condition (18) is incompatible with a regularized imaginary part of the overlap's effective action, at least when a Pauli-Villars like regularization is used (which is the case for the consistent regularization).

\section{Anomalous Divergence of the Chiral Cur- rent.}

The chiral anomaly can also be derived by taking the covariant divergence of the expectation value $j_{\mu}^{a}$ of the chiral current operator $J_{\mu}^{a}$. We shall now derive the anomaly following this procedure for the unregularised overlap. 
To start with, we recall the definition

$$
j_{\mu}^{a}=\left\langle J_{\mu}^{a}\right\rangle=\operatorname{tr}\left[\gamma_{\mu} \tau_{a} S_{F}(x, y)\right]
$$

where $S_{F}(x, y)$ is the propagator for a chiral fermionic field in the presence of an external gauge field. To evaluate (21) in terms of objects defined in the overlap formalism, we just need the overlap definition for the chiral fermion propagator [8]

$$
\begin{gathered}
S(x, y)=\lim _{M \rightarrow \infty} S(x, y ; M) \\
S(x, y ; M)=\frac{1}{M} \frac{\left\langle A+\left|\Psi(x) \Psi^{\dagger}(y)\right| A-\right\rangle}{\langle A+\mid A-\rangle} .
\end{gathered}
$$

By using the definitions presented in section 2, we obtain, after some algebra,

$$
S(x, y ; M)=\frac{1}{2 M}\left\langle x\left|\left(\begin{array}{cc}
1 & -\frac{1}{\hat{\omega}-M} C^{\dagger} \\
-C \frac{1}{\hat{\omega}+M} & 1
\end{array}\right)\right| y\right\rangle,
$$

expression which is valid even if zero modes are present.

We then take the covariant derivative of the current calculated with the finite- $M$ propagator

$$
\begin{gathered}
\left(D_{\mu} j_{\mu}\right)_{a}(x)=\frac{1}{2 M} \lim _{y \rightarrow x} \operatorname{tr}\left[\gamma_{\mu} \tau_{a} D_{\mu}^{x} S(x, y ; M)\right] \\
=\frac{1}{2 M} \lim _{y \rightarrow x} \operatorname{tr}\left\{\left\langle x\left|\tau_{a}\left(\begin{array}{cc}
0 & -C^{\dagger} \\
C & 0
\end{array}\right)\left(\begin{array}{cc}
1 & -\frac{1}{\hat{\omega}-M} C^{\dagger} \\
-C \frac{1}{\hat{\omega}+M} & 1
\end{array}\right)\right| y\right\rangle\right\} \\
=\frac{1}{2 M} \lim _{y \rightarrow x}\left[\left\langle x\left|\operatorname{tr}\left(\tau_{a} \hat{\omega}\right)\right| y\right\rangle-\left\langle x\left|\operatorname{tr}\left(\tau_{a} \tilde{\omega}\right)\right| y\right\rangle\right],
\end{gathered}
$$

where we have defined

$$
\tilde{\omega}=\left(C C^{\dagger}+M^{2}\right)^{\frac{1}{2}} .
$$

We note that a formula that resembles Fujikawa's expression for the anomaly can be derived by simply rewriting (24) as

$$
\left(D_{\mu} j_{\mu}\right)_{a}(x)=\frac{1}{2} \frac{M}{|M|} \int \frac{d^{d} k}{(2 \pi)^{d}} e^{-i k \cdot x} \operatorname{tr}\left[\gamma_{5} \tau_{a} f\left(-\frac{D^{2}}{M^{2}}\right)\right] e^{i k \cdot x}
$$


with $f(x)=\sqrt{1+x}$. It is evident that the result it yields for the anomaly is unregularized, since the would-be regulating function $f$ in this case fails to satisfy the condition $f(\infty)=0$, necessary to make (26) convergent. However, it is worth remarking that the integral over $x$ of (24) is meaningful, since

$$
\int d^{d} x D_{\mu} j_{\mu}(x)=\frac{1}{2} \frac{M}{|M|}\left(n_{+}-n_{-}\right)
$$

where $n_{+}\left(n_{-}\right)$is the number of zero-modes of the operator $C\left(C^{\dagger}\right)$. Hence, the integral of the anomaly is proportional to the index of the operator $C$, as it should be.

This calculation also illustrates what we said at the end of the previous section, since the anomaly (proportional to the variation of the imaginary part) is odd in $M$ and divergent.

\section{Regularization.}

The real part $\operatorname{Re} \Gamma_{\mathcal{O}}$ may be regularized in a gauge-invariant way by introducing in (10) a regulating function depending on the operator $C^{\dagger} C$, which transforms in a gauge-covariant way:

$$
\begin{aligned}
\operatorname{Re} \Gamma_{\mathcal{O}}(A, M, \Lambda) & =-\frac{1}{2} \operatorname{Tr} \log \left[f\left(\frac{C^{\dagger} C}{\Lambda^{2}}\right) \frac{C^{\dagger} C}{C^{\dagger} C+M^{2}}\right] \\
& +\frac{1}{2} \operatorname{Tr} \log \left[f\left(\frac{C_{0}^{\dagger} C_{0}}{\Lambda^{2}}\right) \frac{C_{0}^{\dagger} C_{0}}{C_{0}^{\dagger} C_{0}+M^{2}}\right],
\end{aligned}
$$

where $f$ satisfies the conditions

$$
f(z) \stackrel{z \rightarrow 0}{=} 1 \quad ; f(z) \stackrel{z \rightarrow \infty}{=} f^{\prime}(z) \stackrel{z \rightarrow \infty}{=} \cdots f^{(n)}(z) \stackrel{z \rightarrow \infty}{=} 0
$$

For finite $\Lambda$, the $M \rightarrow \infty$ limit may be taken safely in (28), since conditions (29) assure the convergence of the functional trace.

To regulate the imaginary part is not so straightforward as for the real part. Simple algebraic manipulations in (14) lead to the following convenient expression for the imaginary part,

$$
\operatorname{Im} \Gamma_{\mathcal{O}}(A, M)=\operatorname{Im}\left\{\operatorname{Tr}\left[\log \left(W_{0} I W\right)\right]\right\}
$$


where,

$$
\begin{aligned}
W_{0} & =\hat{\omega}_{0}+M \\
W & =\hat{\omega}+M \\
I & =\left(W W_{0}+C^{\dagger} C_{0}\right)^{-1} C^{\dagger} C_{0}\left(W W_{0}+C^{\dagger} C_{0}\right)^{-1} .
\end{aligned}
$$

It is worth noting that if the property,

$$
\operatorname{Tr}[\log (a b)]=\operatorname{Tr}[\log (a)+\log (b)],
$$

were valid for the factors in (30) then the contribution from the two positivedefinite hermitean factors $\left(\hat{\omega}_{0}+M\right)$ and $(\hat{\omega}+M)$ should cancel in (30) when taking the imaginary part. Regularizing these factors by,

$$
\begin{aligned}
& W_{0}^{r e g}=e^{-W_{0} / \Lambda} W_{0} \\
& W^{r e g}=e^{-W / \Lambda} W,
\end{aligned}
$$

(32) holds 9 . Hence we have,

$$
\operatorname{Im} \bar{\Gamma}_{\mathcal{O}}(A, M)=\operatorname{Im}\{\operatorname{Tr}[\log \mathrm{I}]\} .
$$

The left hand side of (34) has not yet been regularized. This task is rendered difficult since the non-hermitean operator $C^{\dagger} C_{0}$ is not even normal. Hence we can not assert whether it has a well defined eigenvalue problem or not. In this work we will only look for a perturbatively well-defined version of (34). It turns out that the following regularization works in the above sense,

$$
\begin{aligned}
\operatorname{Im} \bar{\Gamma}_{\mathcal{O}}^{r e g}(A, M)= & \operatorname{Im}\left\{\operatorname { T r } \left[\operatorname { l o g } \left(\varphi\left(\frac{\mathrm{C}^{\dagger} \mathrm{C}_{0}}{\Lambda^{2}}\right)\left(\mathrm{WW}_{0}+\mathrm{C}^{\dagger} \mathrm{C}_{0}\right)^{-1}\right.\right.\right. \\
& \left.\left.\left.C^{\dagger} C_{0}\left(W W_{0}+C^{\dagger} C_{0}\right)^{-1}\right)\right]\right\}
\end{aligned}
$$

where $\varphi$ satisfies conditions analogous to (29) for $f$, and its explicit form depends on the spacetime dimension. It is convenient to pass from (35) to

${ }^{3}$ Using the Backer-Haussdorff relation it is simple to show that (32) is valid if,

$$
\operatorname{Tr}([\log a, \log b]+\text { additional nested commutators })=0
$$

by means of theorem VI.25 of Ref.([1] ) this is assured if one of the factors of the commutators is trace class and the other is bounded. This is so when employing the regularized operators (33) in this first regularized version $\operatorname{Im} \bar{\Gamma}_{\mathcal{O}}(A, M)$ of $(30)$. 
a simplified version which however yields the same results when $M$ is taken to be infinite. The simplified formula follows from the observation that, for a finite $\Lambda$, one can take $M$ to be much larger than $\Lambda$ and thus than all the momentum scales in the problem. It is then possible to make a large- $M$ expansion, which to leading order amounts to:

$$
\hat{\omega} \simeq \hat{\omega}_{0} \simeq|M| \quad, \quad W W_{0} \simeq 4 M^{2}
$$

Thus the regularized imaginary part is approximated by

$$
\begin{aligned}
\operatorname{Im} \bar{\Gamma}_{\mathcal{O}}^{r e g}(A, M)= & \operatorname{Im}\left\{\operatorname { T r } \left[\operatorname { l o g } \left(\varphi\left(\frac{\mathrm{C}^{\dagger} \mathrm{C}_{0}}{\Lambda^{2}}\right)\left(4 \mathrm{M}^{2}+\mathrm{C}^{\dagger} \mathrm{C}_{0}\right)^{-1}\right.\right.\right. \\
& \left.\left.\left.C^{\dagger} C_{0}\left(4 M^{2}+C^{\dagger} C_{0}\right)^{-1}\right)\right]\right\} .
\end{aligned}
$$

Incidentally, this expression is tantamount to making the replacements $\mathcal{C} \rightarrow$ $C /(2 M), \mathcal{C}_{0} \rightarrow C_{0} /(2 M)$ in (35). This can be understood from the fact that in a polar decomposition both $\mathcal{C}$ and $C$ have the same unitary part. This part should be the only important one in the determination of the imaginary part at large $M$. We have also explicitly verified that an expansion in powers of $A_{\mu}$ of the finite- $M$ expression (34) has the same superficial degree of divergence as the one approximated for large values of $M$.

An unpleasant feature of expression (37) is that it depends on the two masses $M$ and $\Lambda$, which should eventually tend to infinity. This introduces the problem of deciding an order for taking those limits. We discard the order where $\Lambda$ is taken to infinity before $M$, since we know that we then get an expression where $\varphi$ is replaced by one, which is not regularized. The natural order seems to be the one where $M$ is taken to infinity first, with the proviso that the form of $\varphi$ should be chosen in order to render the imaginary part finite even when $M \rightarrow \infty$ (what can always be done). When this limit has been taken, we are led to an expression of the form

$$
\operatorname{Im} \bar{\Gamma}_{\mathcal{O}}^{r e g}(A)=\operatorname{Im}\left\{\operatorname{Tr}\left[\log \left(\varphi\left(\frac{\mathrm{C}^{\dagger} \mathrm{C}_{0}}{\Lambda^{2}}\right) \frac{\mathrm{C}^{\dagger} \mathrm{C}_{0}}{\Lambda^{2}}\right)\right]\right\}
$$

where the factor $\frac{1}{\Lambda^{2}}$ has been introduced to render the argument of the logarithm dimensionless (it does not affect the imaginary part, though).

Next we apply the previously discussed concepts to the two-dimensional non-Abelian case in perturbation theory. It is straightforward to see that the 
real part is Pauli-Villars regulated for a finite $M$. The perturbative series for Re $\Gamma_{\mathcal{O}}$ can be put as

$$
\operatorname{Re} \Gamma_{\mathcal{O}}=-\frac{1}{2} \sum_{r=1}^{\infty} \frac{(-1)^{r-1}}{r} \sum_{s=0}^{1} C_{s} \operatorname{Tr}\left\{\left[\left(-\partial^{2}+M_{s}^{2}\right)^{-1} V\right]^{r}\right\}
$$

where

$$
V=C_{0}^{\dagger} \sigma \cdot A-\sigma^{\dagger} \cdot A C_{0}-\sigma^{\dagger} \cdot A \sigma \cdot A
$$

and we have used the property $C_{0}^{\dagger} C_{0}=-\partial^{2}$. To realize that (39) is regulated, we just note that the superficial degree of divergence of the $(s=0$ or $s=1)$ term of order $r$ is $\omega_{r}=2-r$. Hence all the terms with $r>2$ converge (even for an infinite $M$ ). We only need to consider the terms with $r=1$ and $r=2$, which shall be denoted by $\gamma_{1}$ and $\gamma_{2}$, respectively. $\gamma_{1}$ may be written as

$$
\gamma_{1}(A, M)=-\frac{1}{2} \int \frac{d^{2} k}{(2 \pi)^{2}} \frac{M^{2}}{k^{2}\left(k^{2}+M^{2}\right)} \sigma_{\mu}^{\dagger} \sigma_{\nu} \int d^{2} x A_{\mu}^{a}(x) A_{\mu}^{a}(x),
$$

which is obviously convergent. The second order term $\gamma_{2}$ is given by

$$
\begin{gathered}
\gamma_{2}=\frac{1}{4} \sum_{s=0}^{1} C_{s} \operatorname{Tr}\left\{\left[\left(-\partial^{2}+M^{2}\right)^{-1}\left(C_{0}^{\dagger} \sigma \cdot A-\sigma^{\dagger} \cdot A C_{0}\right)\left(-\partial^{2}+M^{2}\right)^{-1}\right.\right. \\
\left.\left.\left(C_{0}^{\dagger} \sigma \cdot A-\sigma^{\dagger} \cdot C_{0}\right)\right]+\left[\left(-\partial^{2}+M^{2}\right)^{-1} \sigma^{\dagger} \cdot A \sigma \cdot A\left(-\partial^{2}+M^{2}\right)^{-1} \sigma^{\dagger} \cdot A\right]\right\} .
\end{gathered}
$$

To make the degree of divergence explicit, we take the trace in momentum space. One sees that it contains the two momentum integrals

$$
\begin{aligned}
& I_{1 \mu \nu}=\int \frac{d^{2} k}{(2 \pi)^{2}} \sum_{s=0}^{1} C_{s}\left\{\frac{k_{\mu}(k+p)_{\nu}}{\left(k^{2}+M_{s}^{2}\right)\left[(k+p)^{2}+M_{s}^{2}\right]}\right\} \\
& I_{2}=\int \frac{d^{2} k}{(2 \pi)^{2}} \sum_{s=0}^{1} C_{s}\left\{\frac{1}{\left(k^{2}+M_{s}^{2}\right)\left[(k+p)^{2}+M_{s}^{2}\right]}\right\} .
\end{aligned}
$$

A simple analysis of the large momentum behaviour of these integrals shows that they are convergent, with superficial degrees of divergence $\omega=-2$ and $\omega=-4$ for $I_{1}$ and $I_{2}$, respectively. This completes the proof of the finiteness of the real part for the two dimensional case. 
Regarding the imaginary part, the form of the regulating function which is used to make it finite can always be chosen in a Pauli-Villars like fashion, which corresponds to the consistent anomaly [1]. It is worth noting, however, that if one accepts a definition of the imaginary part where the term $A$ defined in (15) is divergent, but the divergencies cancell when taking its imaginary part, no regularization is needed, at least in two dimensions. This, however, is a very special way of evaluating the imaginary part, and one can say that amounts to a kind of 'symmetric' regularization. For the Abelian case in two dimensions, this evaluation yields, after taking the limit $M \rightarrow \infty$ :

$$
\operatorname{Im} \bar{\Gamma}_{\mathcal{O}}(A)=\frac{1}{4 \pi} \int d^{2} x \partial \cdot A \frac{1}{\partial^{2}} \epsilon_{\mu \nu} \partial_{\mu} A_{\nu}
$$

which yields the right value for the anomaly when taking the gauge variation.

The results about regularization are summarized in the table bellow. They depend on the dimension and on whether we are considering the real or the imaginary part.

\section{Real}

$$
\begin{array}{cc}
d=2 & \begin{array}{c}
\text { Converges with } f=1 \\
\text { Regularization not necessary } \\
\text { Necessary. Converges for } \\
\text { adequate choice of } f .
\end{array}
\end{array}
$$

Imaginary

Converges with $f=1$

However neither $A$ nor $A^{\dagger}$ converge.

Necessary.Converges at least perturbatively for adequate $f$.

\section{Anomalies}

We start from expression (37). Its gauge variation is given by,

$$
\delta \operatorname{Im} \bar{\Gamma}_{\mathcal{O}}^{r e g}(A)=-\frac{1}{2 i}\left\{\operatorname{Tr}\left[\varphi\left(\frac{C_{0}^{\dagger} C}{\Lambda^{2}}\right) \frac{C_{0}^{\dagger} \delta C}{\Lambda^{2}}\right]-\operatorname{Tr}\left[\varphi\left(\frac{C_{0} C^{\dagger}}{\Lambda^{2}}\right) \frac{C_{0} \delta C^{\dagger}}{\Lambda^{2}}\right]\right\}
$$

where,

$$
\varphi(x)=f^{-1}(x) \frac{d f(x)}{d x}+\frac{1}{x},
$$

rewriting this last expression in Dirac space we are lead to,

$$
\delta \operatorname{Im} \bar{\Gamma}_{\mathcal{O}}^{r e g}(A)=\frac{1}{2 i \Lambda^{2}} \operatorname{Tr}\left\{\varphi\left(-\frac{\not \supset \not D}{\Lambda^{2}}\right) \gamma_{5} \not \partial[\not D, \omega]\right\}
$$


$\omega$ being, as before, the infinitesimal parameter of the gauge transformation. Defining $\mathcal{A}_{a}(x)$ by,

$$
\delta \phi_{\text {reg }}(A, \Lambda)=\int d^{d} x \omega_{a}(x) \mathcal{A}_{a}(x)
$$

we get,

$$
\mathcal{A}_{a}(x)=D_{\mu}^{a b} J_{\mu}^{b}(x)
$$

with,

$$
J_{\mu}^{a}(x)=-\frac{1}{2 i \Lambda^{2}} \operatorname{Tr}\left\langle x\left|\varphi\left(-\frac{\not \supset \not D}{\Lambda^{2}}\right) \gamma_{5} \not \partial \gamma_{\mu} \tau^{a}\right| x\right\rangle,
$$

for $\Lambda^{2} \rightarrow \infty$ we obtain the unregulated expression,

$$
J_{\mu}^{a}(x)=-\frac{1}{2 i} \operatorname{Tr}\left\langle x\left|-\frac{1}{\not \supset \not D} \gamma_{5} \not \partial \gamma_{\mu} \tau^{a}\right| x\right\rangle
$$

showing that (48) corresponds to a regularization of the first order variation of the chiral determinant, therefore leading to a consistent anomaly.

We conclude that,

(i) When regularized the overlap claim (8) is correct.

(ii) The parameter $M$ can be interpreted as a regularization parameter only for the real part of the overlap effective action $\left(\Gamma_{\mathcal{O}}\right)$ in $d=2$.

(iii) The regularized overlap leads, as it should be, to consistent anomalies for the gauge variation of $\Gamma_{\mathcal{O}}$.

\section{Acknowledgements.}

We acknowledge conversations with Profs. R. Iengo and S. Randjabar-Daemi. C. D. F. thanks Fundación Antorchas for financial support. R. C. T. does the same with the ICTP. 


\section{References}

[1] For an excellent review see, for example, R. D. Ball, Phys. Rep. 182, numbers 1 and 2 (1989).

[2] D. B. Kaplan, Phys. Lett. B288 342 (1992).

[3] S.A. Frolov and A.A. Slavnov, Phys.Lett. B309 344 (1993).

[4] A.A. Slavnov, Phys.Lett. B348 553 (1995).

[5] G. t'Hooft.

[6] R. Narayanan and H. Neuberger, Nucl. Phys. B443:305-385 (1995). See also: R. Narayanan, H. Neuberger and P. Vranas, hep-lat/9509046; R. Narayanan and H. Neuberger, hep-lat/9509047; H. Neuberger, hep-lat/9511001.

[7] S. Randjbar-Daemi and J. Strathdee, Phys. Lett. B348, 543 (1995).

[8] S. Randjbar-Daemi and J. Strathdee, Nucl. Phys. B443 386 (1995)

[9] S. Randjbar-Daemi and J. Strathdee, Phys. Rev. D51, 6617 (1995).

[10] S. Randjbar-Daemi and J. Strathdee, Nucl. Phys. B466, 335 (1996).

[11] M. Reed and B. Simon, Functional Analysis, Academic Press, 1972.

[12] C.D. Fosco, Int. J. Mod. Phys. A11 3987 (1996). 